\section{OP-38 BUILDING CAPACITY OF LOCAL MEDIA TO FACILITATE INTERSECTORAL ACTION FOR HEALTH IN THE SUNDARBANS, WEST BENGAL}

Upasona Ghosh, Shibaji Bose, Rittika Bramhachari, Sabyasachi Mondol. IIHMR Institute of Health Management Research, Kolkata (West Bengal), India

\subsection{6/bmjgh-2016-EPHPabstracts.38}

Background In this paper, we describe preliminary findings from an on-going capacity-building project that engages local media as key facilitator to achieve convergence across various health system policy initiatives in a disadvantaged region, the Indian Sundarbans, West Bengal. The purpose is to understand whether and how local media could be used to push for intersectoral action based on the health demands of the community. Subsequently, the project aims at building capacity of the local media for effective advocacy and context-specific facilitation.

Methods In the first stage, we performed a detailed scan of news stories from five purposively chosen local print media spanning one-year duration. We simultaneously conducted in-depth interviews with community representatives and local media journalists/owners to explore the role of local media in Sundarbans' health system. In the on-going second stage, we conduct participatory training workshops with local media associations to orient them on how to better represent the community's health demands. We provide mentorship to journalists as and when required.

Findings News scan and interviews with community representatives and local media journalists/owners on the one hand identified many a range of knowledge gaps among journalists and media owners regarding community's specific health demands. Community representatives on the other hand see local media as a potential resource to influence community-level policy dynamics and to achieve intersectoral convergence.

The identified knowledge gaps, as reflected in media reportage on health, persists in several areas: (1) representation of community's health demands; (2) details about basic health system elements; (3) links health services with contextual determinants; and (4) use of research findings while making a case. Media reportage on health was predominantly on issues related to curative care $(68 \%)$ and much less about preventive care and health promotion. Except for a few news stories constituting mere $3.4 \%$ of health coverage that were about access and mid-day meal schemes (under Integrated Child Development Scheme), the media did not highlight systemic factors and policy determinants to meet the community's context-specific health demands.

On-going news scan, following a first round of capacity building of local media associations, reveals that health reportage is gradually increasing. A total $2.3 \%$ increase in health reportage has been observed in last six-months. Research findings are being used either as standalone stories or to build a case. There has been demand from journalists' associations from other blocks (geographic units) for similar capacity-building initiatives. Media associations have linked up with the health department and other related departments engaged in improving health services. They also have linked up with community-based organisations for outdoor dissemination of health messages among the community through an edutainment format.

Discussion There is need for planned and sustained capacity building of local media for them to play a larger role in facilitating intersectoral action on health. The community's faith in local media provides an opportunity to act as a conduit for policy actors on intersectoral action. A structured and sustained knowledge sharing with the media on health systems would create an enabling health policy environment for uptake of evidence-informed research, and eventually for better health.

Our study has potential to add to the existing knowledge in areas of health system strengthening as well as media and communication research. We hope it encourages researchers to consider the integrating role of media and communication in health system studies as related to intersectoral action for health, as well as uptake of community knowledge and research evidence into policy-making processes.

Grant funding (to Future Health Systems Research Consortium by DfID - Department for International Development, London, UK) for research but no other competing interests. 